\title{
Desigualdades regionais nas relações de trabalho e qualificação profissional de cirurgiões-dentistas atuantes na Atenção Básica
}

Letícia Rosa*; Ana Elisa Ribeiro**; Manoelito Ferreira Silva-Junior***; Márcia Helena Baldani****

* Estudante de Odontologia, Universidade Estadual de Ponta Grossa

** Doutoranda em Odontologia, Universidade Estadual de Ponta Grossa

*** Professor Colaborador Adjunto, Universidade Estadual de Ponta Grossa

**** Professora Associada, Departamento de Odontologia, Universidade Estadual de Ponta Grossa

Recebido em 20/06/2020. Aprovado em 11/07/2021.

\begin{abstract}
RESUMO
O estudo transversal objetivou comparar as relações de trabalho e qualificação profissional de cirurgiões-dentistas atuantes na Atenção Básica nas regiões geográficas brasileiras. Utilizou-se dados do Módulo VI da avaliação externa do $2^{\circ}$ ciclo do Programa Nacional do Acesso e da Qualidade da Atenção Básica (PMAQ-AB). A comparação entre regiões foi realizada pelo teste qui-quadrado $(\mathrm{p}<0,05)$ e análises descritivas. Das 17.117 Equipes de Saúde Bucal avaliadas, a maioria dos cirurgiões dentistas era Servidor Público Estatutário $(44,1 \%)$, sendo, este número, maior na região Sul $(63,6 \%)$ e menor no Sudeste $(37,8 \%)$, com ingresso por concurso público $(49,6 \%)$, sendo maior no Sul $(79,5 \%)$ e menor no Nordeste $(40,9 \%)$. Em relação à qualificação profissional, $(73,1 \%)$ possuíam formação complementar e dentre estes, a maioria tem especialização em outras áreas que não Saúde Coletiva $(53,2 \%)$, sendo maior no Norte $(59,3 \%)$ e menor no Nordeste $(50,4 \%)$. Os especialistas em Saúde da Família ou Saúde Pública/Saúde Coletiva (26,7\% e 18,7\%) respectivamente foram mais frequentes no Sudeste $(38,7 \%$ e $21,4 \%)$ e menor no Norte $(11,4 \%$ e $14,9 \%)$ respectivamente, para todas o valor de " $p$ " foi $(\mathrm{p} \leq 0.001)$. Portanto, houve desigualdades regionais nas relações de trabalho e qualificação profissional no Brasil, e por isso, políticas indutoras para fixação dos profissionais e sua qualificação precisam ser reforçadas.
\end{abstract}

Descritores: Saúde Bucal. Recursos Humanos em Saúde. Qualificação Profissional. 


\section{INTRODUÇÃO}

A história da saúde bucal brasileira é caracterizada por ausência de políticas públicas consistentes e foi, por muito tempo, marcada por práticas de saúde excludentes e mutiladoras ${ }^{1}$. Em 1988, com a promulgação da atual Constituição Federal, a saúde se tornou um direito da população brasileira, e foi instituído o Sistema Único de Saúde (SUS) que apresenta como princípios doutrinários, a universalidade, a integralidade e a equidade ${ }^{2}$. No entanto, a saúde bucal teve sua inserção ampliada nos serviços públicos de saúde apenas anos mais $\operatorname{tarde}^{3,4}$.

Sendo assim, no intuito de garantir a universalidade da atenção, no final do ano 2000 foi lançada a portaria do Ministério da Saúde (MS) 1.444, que incluiu a Equipe de Saúde Bucal (ESB) na Estratégia de Saúde da Família (ESF). Com isso, buscava-se a reorganização dos serviços odontológicos na atenção básica $(\mathrm{AB})$ e melhoria nos indicadores epidemiológicos de saúde bucal da população brasileira ${ }^{3}$. Em 2004, através da atual Política Nacional de Saúde Bucal, mais um passo foi dado para a integralidade, através da ampliação dos serviços em atenção básica e disponibilidade em nível secundário e terciário 4 .

A expansão do número de equipes de saúde bucal e a inclusão de serviços especializados tornaram o SUS o maior empregador de cirurgiõesdentistas (CD) do Brasil na atualidade ${ }^{5}$. No entanto, há distribuição heterogênea do número de profissionais entre os estados brasileiros, principalmente decorrente de uma aglomeração de faculdades de Odontologia na região centro-sul do país $^{6}$. Isso pode acarretar dificuldades de gestão e fixação destes profissionais e, por consequência, diferenças regionais na alocação dos recursos humanos no SUS. Sendo assim, os diferentes vínculos trabalhistas disponibilizados no sistema público de saúde podem prejudicar a estabilidade dos trabalhadores, e assim, a qualidade dos serviços oferecidos à população ${ }^{7}$.
Ao ser inserido na ESF, o CD com os demais componentes da equipe (auxiliares e técnicos em saúde bucal), precisou adaptar-se a um novo processo de trabalho, o qual anteriormente era desenvolvido com vistas a uma prática predominantemente privatista, curativista e biologicista $^{3}$. No intuito de responder às necessidades de saúde da população usuária dos serviços odontológicos na ESF, a capacitação profissional foi estimulada com o intuito de fomentar processos de formação vinculados à Educação Permanente em Saúde. Desde a década de 90, o MS vem investindo na qualificação da atenção básica, através de cursos de atualização, aperfeiçoamento e pós-graduação lato sensu, como Especialização e Residência Multiprofissional em Saúde da Família 8 .

Um dos principais objetivos da Política Nacional de Educação Permanente em Saúde (PNEPS) é fortalecer o SUS sendo uma estratégia de formação e capacitação para o desenvolvimento do trabalho na saúde9. A disponibilidade da Educação Permanente (EP) se materializa em um forte instrumento de gestão do trabalho, justificando seu valor na melhoria da qualificação do processo de trabalho dos profissionais e da equipe como um todo ${ }^{10}$.

Como todo processo de trabalho, a atenção em saúde deve ser monitorada e avaliada para que melhorias possam ser implementadas englobando desde as dimensões de produtividade, satisfação, realização pessoal dos profissionais e usuários, economia de meios e otimização de recursos. Com esta finalidade, o SUS conta atualmente com o Programa Nacional de Melhoria do Acesso e da Qualidade da Atenção Básica (PMAQ-AB), que mensura vários eixos estruturantes da $\mathrm{AB}$, incluindo os vínculos profissionais e a formação profissional, que podem interferir no processo de trabalho melhorando a qualidade da atenção ${ }^{11,12}$. Desse modo, o objetivo deste estudo foi identificar desigualdades regionais nas relações de trabalho e 
qualificação profissional de cirurgiões-dentistas atuantes na $\mathrm{AB}$ no Brasil, segundo dados do segundo ciclo do PMAQ-AB.

\section{METODOLOGIA}

O estudo, caracterizado como quantitativo, transversal e analítico, foi realizado com dados secundários do Módulo VI do instrumento de avaliação externa do $2^{\circ}$ ciclo (2013-2014) do PMAQ-AB, disponibilizados pelo Ministério da Saúde.

O universo amostral consistiu nas ESB da $\mathrm{AB}$ (ESB da ESF ou parametrizadas) que aderiram e receberam avaliação externa do $2^{\circ}$ Ciclo do PMAQ-AB. Foram selecionadas variáveis do Módulo VI do instrumento de coleta de dados, que constava de entrevista com profissional da ESB (CD ou técnico/auxiliar em saúde bucal) e verificação de documentos na unidade de saúde. $\mathrm{Na}$ composição da análise do presente estudo, apenas os formulários respondidos pelos $\mathrm{CD}$ foram incluídos, visto que o objetivo do estudo se referiu e estes profissionais.

A avaliação externa foi realizada de forma multicêntrica, a cargo de instituições de ensino superior dos 27 estados brasileiros, as quais coordenaram equipes de entrevistadores calibrados que deveriam coletar os dados junto aos profissionais utilizando formulários validados, registrar as respostas em tablets e analisar os documentos comprobatórios quando o instrumento assim o solicitava. Aos participantes, era apresentado um termo de consentimento livre e esclarecido e lhes era assegurado o direito de recusa em participar.

O Módulo VI era composto por 230 questões subdivididas por temas. As subdivisões de VI-3 a VI-6 foram as utilizadas no presente estudo e correspondiam aos seguintes temas: VI-3 Formação e qualificação dos profissionais da Equipe de Saúde Bucal; VI-4 Vínculo; VI-5 Plano de Carreira; VI-6 Educação Permanente no processo de qualificação das ações desenvolvidas. Sendo assim, um total de 32 questões foram analisadas e incluídas.

As variáveis investigadas foram divididas em dois eixos (quadro 1): a) relações de trabalho, que procuraram representar os vínculos trabalhistas e as características dos agentes contratantes; b) formação e qualificação profissional, que identificaram as ações de educação continuada e permanente ofertadas às ESB (ESF ou parametrizadas). Os resultados foram comparados entre as regiões do Brasil (Norte, Nordeste, CentroOeste, Sudeste e Sul).

Os dados obtidos foram analisados no Statistical Package for the Social Sciences (SPSS) 20.0 (IBM, Armonk, NY, EUA), e apresentados em frequências absolutas (n) e relativas (\%). As associações das variáveis estudadas (desfechos) e as regiões do Brasil (variável independente) foram realizadas utilizando o teste qui-quadrado $(\mathrm{p}<0,05)$. Entre as variáveis em que as opções de respostas eram 'Sim' ou 'Não', apesar das tabelas do banco de dados do PMAQ-AB apresentarem apenas a categoria 'Sim', a categoria 'Não' também foi considerada na análise.

A avaliação externa do segundo ciclo do PMAQ-AB foi coordenada pela Escola Nacional de Saúde Pública/Fundação Oswaldo Cruz (Fiocruz) e foi aprovado pelo Comitê de Ética em Pesquisa institucional sob o número 02040212.1.0000.5240 e os dados são disponibilizados como de domínio público.

\section{RESULTADOS}

A amostra inicial do estudo era composta por 18.115 ESB que responderam ao módulo VI do questionário, sendo 998 excluídas por terem sido respondidos por Auxiliar ou Técnico em Saúde Bucal, restando 17.117 ESB incluídas no estudo.

A maioria dos $\mathrm{CD}$ apresentava vínculo empregatício do tipo servidor público estatutário $(44,1 \%)$, com maior prevalência no Sul $(63,6 \%)$ 
Quadro 1. Variáveis e categorias de análise

\begin{tabular}{|c|c|c|}
\hline Eixo & Variável & Categorias \\
\hline \multirow{8}{*}{ 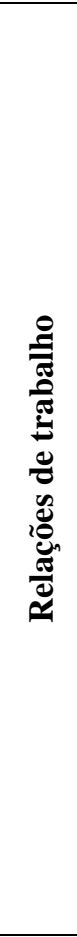 } & Agente contratante & $\begin{array}{l}\text { Administração Direta; Consórcio Intermunicipal de } \\
\text { Direito Público; Consórcio Intermunicipal de Direito } \\
\text { Privado; } \\
\text { Fundação Pública de Direito Público; Fundação Pública } \\
\text { de Direito Privado; Organização Social (OS); } \\
\text { Organização da Sociedade Civil de Interesse Público } \\
\text { (OSCIP); Entidade Filantrópica; Organização não } \\
\text { Governamental (ONG); Empresa; Cooperativa; Outros }\end{array}$ \\
\hline & Tipo de vínculo & $\begin{array}{l}\text { Servidor Público Estatutário; Cargo comissionado; } \\
\text { Contrato temporário pela administração pública regido } \\
\text { por legislação especial (municipal/estadual/federal); } \\
\text { Contrato temporário por prestação de serviço; } \\
\text { Empregado público - Consolidação das Leis do Trabalho } \\
\text { (CLT); Contrato CLT; Autônomo; Outros }\end{array}$ \\
\hline & Forma de ingresso & $\begin{array}{l}\text { Concurso público; Seleção pública; Indicação; Outra } \\
\text { forma }\end{array}$ \\
\hline & Tem plano de carreira & \multirow{5}{*}{ Sim/Não } \\
\hline & Progressão por antiguidade & \\
\hline & $\begin{array}{l}\text { Progressão por avaliação de desempenho e/ou } \\
\text { desenvolvimento (mérito) }\end{array}$ & \\
\hline & Progressão por titulação/ formação profissional & \\
\hline & $\begin{array}{l}\text { Incentivo, gratificação, prêmio financeiro por } \\
\text { desempenho. }\end{array}$ & \\
\hline \multirow{15}{*}{ 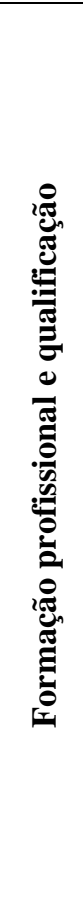 } & Especialização em Saúde da Família & \multirow{12}{*}{ Sim/Não } \\
\hline & Especialização em Saúde Pública/ Saúde Coletiva. & \\
\hline & Outra especialização & \\
\hline & Residência em Saúde da Família & \\
\hline & Residência em Saúde Pública/ Saúde Coletiva & \\
\hline & Outra residência & \\
\hline & Mestrado em Saúde da Família & \\
\hline & Mestrado em Saúde Pública/ Saúde Coletiva & \\
\hline & Outro mestrado & \\
\hline & Doutorado em Saúde da Família & \\
\hline & Doutorado em Saúde Pública/ Saúde Coletiva & \\
\hline & Outro doutorado & \\
\hline & $\begin{array}{l}\text { Quais dessas ações de Educação Permanente (EP) a } \\
\text { ESB participa: }\end{array}$ & $\begin{array}{l}\text { Seminários; Mostras; Oficinas; Grupos de Discussão; } \\
\text { Cursos presenciais; Telessaúde; Rede Universitária de } \\
\text { Telemedicina (RUTE); Universidade Aberta do SUS } \\
\text { (UNASUS); Cursos de Educação à Distância; Troca de } \\
\text { experiência; Tutoria/preceptoria; UBS recebe alunos de } \\
\text { graduação, especialização, residentes entre outros }\end{array}$ \\
\hline & $\begin{array}{l}\text { Quais profissionais da ESB estão envolvidos nas ações } \\
\text { de EP }\end{array}$ & $\begin{array}{l}\text { Apenas o Cirurgião Dentista; Apenas o Auxiliar e/ou } \\
\text { Técnico em Saúde Bucal; ESB completa }\end{array}$ \\
\hline & $\begin{array}{l}\text { Essas ações de EP contemplam as demandas e } \\
\text { necessidades da Equipe }\end{array}$ & Sim/Não \\
\hline
\end{tabular}

e menor no Sudeste $(37,8 \%)$. O segundo tipo de vínculo com relevância analisado foi o contrato temporário por prestação de serviço $(20,4 \%)$, a região com a maior quantidade desse tipo de vínculo foi o Nordeste $(30,1 \%)$ e a região com menor foi o Sul $(3,0 \%)$. A principal forma de ingresso no serviço público entre os $\mathrm{CD}$ foi por concurso público $(49,6 \%)$, sendo maior na região Sul $(79,5 \%)$ e menor no Nordeste $(40,9 \%)$. A forma de ingresso por indicação também 
apresentou alta prevalência $(23,1 \%)$ sendo as regiões Nordeste e Norte as com maiores porcentagens $(33,5 \%)$ e $(30,3 \%)$, respectivamente e o Sul e Sudeste as menores $(4,4 \%)$ e $(16,9 \%)$, respectivamente (tabela 1 ).

$\mathrm{O}$ principal agente contratante foi a administração direta (82,5\%), sendo a maior proporção na região Norte $(90,7 \%)$ e menor na Sudeste $(73,1 \%)$. Dos 16.642 CD que responderam a questão sobre plano de carreira, $21 \%$ afirmaram ter plano, sendo a região Sul com maior prevalência $(40,4 \%)$ e o Nordeste a menor $(12,4 \%)$. Dentre os tipos de progressão analisados a progressão por antiguidade foi a que mais se destacou em âmbito nacional $(87,9 \%)$ (tabela 1$)$.

Em relação à qualificação profissional, $73,1 \%$ responderam que possuem ou estão realizando algum tipo de formação complementar. $\mathrm{Na}$ análise incluindo apenas os $\mathrm{CD}$ que realizaram/estão realizando formação complementar, a pós-graduação latu sensu com maior prevalência foi especialização em outra área que não a Saúde da Família/Saúde Coletiva/Saúde Pública $(53,2 \%)$, sendo maior no Norte $(59,3 \%)$ e menor no Nordeste $(50,4 \%)$. Entre os que realizaram pós-graduação strictu sensu, houve maior formação no nível de Mestrado em outra área que não a Saúde da Família/Saúde Coletiva/Saúde Pública $(7,2 \%)$ e foram maior número no Norte $(8,3 \%)$ e menor no Nordeste $(6,5 \%)(\mathrm{p}=0,046)$ (tabela 2).

Em relação aos tipos de ações de EP praticados pelas ESB no Brasil houve maior prevalência em participação do tipo Seminários, Mostras, Oficinas e Grupos de discussão (64,2\%), sendo maior no Sul $(70,6 \%)$ e menor no Norte $(53,5 \%)$. O RUTE-Telemedicina foi a ação menos utilizada $(1,5 \%)$, sendo mais na região Sul $(2,7 \%)$ e menos na região Nordeste $(1,0 \%)$. Nas ações de $\mathrm{EP}$, dentre as equipes que responderam que participaram, 82,0\% das ações eram voltadas para todos os membros da ESB, 14,1\% apresentaram ações apenas para o $\mathrm{CD}$, e 3,9\% incluem apenas o Auxiliar ou Técnico em Saúde Bucal, também com diferenças regionais (tabela 3 ).

Os CD avaliaram que as ações de EP contemplam as demandas e necessidades da Equipe $(74,4 \%)$, sendo maior na região Sudeste $(77,6 \%)$ e menor no Norte $(63,7 \%) \quad(\mathrm{p} \leq 0,001)$ (figura 1).

\section{DISCUSSÃO}

A maioria dos CD participantes do PMAQ$\mathrm{AB}$ possuiam vínculos de trabalho considerados formais pelo MS e ingressaram no emprego via concurso público, sendo a administração direta o principal agente contratante. No entanto, a maioria dos profissionais não tem plano de carreira e os tipos de progressão prevalentes são por antiguidade e titulação/formação profissional. Esses achados coincidem com estudo realizado com médicos atuantes no SUS no I e II ciclos da avaliação externa do PMAQ-AB ${ }^{13}$, ou seja, faz parte da empregabilidade do setor de saúde nos municípios, e não atrelado apenas ao tipo de profissional vinculado ao SUS.

O Nordeste, região que apresentou o maior número de ESB participantes na avaliação externa do II ciclo do PMAQ-AB, foi o local que apresentou menor proporção de vínculos formais de trabalho e admissão via concurso público. Nessa região ocorreu a maior prevalência da forma de ingresso por indicação. Em estudo prévio no Nordeste, foram observados melhores vínculos de trabalho para os Agentes Comunitários de Saúde ${ }^{14}$, o que pode demonstrar maior facilidade de fixação de profissionais em nível fundamental e médio, o que não acontece entre profissionais de nível superior, aspecto também visualizado no estado do Paraná, onde os piores tipos de vínculos estão associados à formação de nível superior ${ }^{15}$. Algumas consequências dessa flexibilização das relações de trabalho, tais como a insatisfação do trabalhador, a depreciação do compromisso do 
Tabela 1. Vínculos de trabalho das Equipes de Saúde Bucal participantes da avaliação externa do segundo ciclo do PMAQ-AB nas regiões do Brasil, 2013-2014

\begin{tabular}{|c|c|c|c|c|c|c|c|}
\hline \multirow{3}{*}{ Variável } & \multirow{2}{*}{$\begin{array}{c}\text { Brasil } \\
(\mathrm{n}=\mathbf{1 6 9 8 2})\end{array}$} & \multicolumn{5}{|c|}{ Região geográfica } & \multirow{3}{*}{ p-valor } \\
\hline & & $\begin{array}{c}\text { Sul } \\
(\mathrm{n}=\mathbf{2 4 6 7})\end{array}$ & $\begin{array}{c}\text { Sudeste } \\
(n=4711)\end{array}$ & $\begin{array}{l}\text { Centro-Oeste } \\
(\mathbf{n}=\mathbf{1 5 0 8})\end{array}$ & $\begin{array}{l}\text { Nordeste } \\
(n=7143)\end{array}$ & $\begin{array}{c}\text { Norte } \\
(\mathbf{n}=\mathbf{1 1 5 3})\end{array}$ & \\
\hline & n $(\%)$ & n (\%) & n (\%) & n $(\%)$ & n $(\%)$ & n $(\%)$ & \\
\hline \multicolumn{8}{|l|}{ Tipo de vínculo } \\
\hline Servidor público estatutário/ empregado público CLT & $8587(50,5)$ & $2034(82,4)$ & $2139(45,4)$ & $866(57,5)$ & $3046(42,7)$ & $502(43,5)$ & \multirow[t]{3}{*}{$<0,001$} \\
\hline $\begin{array}{l}\text { Contrato temporário: por administração pública regido por legislação } \\
\text { especial, por prestação de serviço ou CLT }\end{array}$ & $7844(46,2)$ & $396(16,1)$ & $2464(52,3)$ & $583(38,7)$ & $3797(53,2)$ & $604(52,4)$ & \\
\hline Cargo comissionado, autônomo e outros. & $551(3,2)$ & $37(1,5)$ & $108(2,2)$ & $59(3,9)$ & $300(4,2)$ & $47(4,1)$ & \\
\hline \multicolumn{8}{|l|}{ Forma de ingresso } \\
\hline Concurso público & $8494(49,6)$ & $1970(79,5)$ & $2217(46,6)$ & $864(57,3)$ & $2946(40,9)$ & $497(42,7)$ & \multirow[t]{4}{*}{$<0,001$} \\
\hline Seleção pública & $2792(16,3)$ & $290(11,7)$ & $1257(26,4)$ & $225(14,9)$ & $879(12,2)$ & $141(12,1)$ & \\
\hline Indicação & $3951(23,1)$ & $109(4,4)$ & $807(16,9)$ & $269(17,8)$ & $2413(33,5)$ & $353(30,3)$ & \\
\hline Outra forma & $1880(11,0)$ & $109(4,4)$ & $481(10,1)$ & $151(10,0)$ & $966(13,4)$ & $173(14,9)$ & \\
\hline \multicolumn{8}{|l|}{ Agente contratante } \\
\hline Administração direta & $14013(82,5)$ & $2124(86,2)$ & $3441(73,1)$ & $1196(79,6)$ & $6202(86,8)$ & $1050(90,7)$ & \multirow[t]{5}{*}{$<0,001$} \\
\hline Consórcio intermunicipal de direito público ou privado & $365(2,1)$ & $32(1,3)$ & $55(1,1)$ & $66(4,4)$ & $187(2,6)$ & $25(2,2)$ & \\
\hline Fundação pública de direito público ou privado & $817(4,8)$ & $210(8,5)$ & $317(6,8)$ & $86(5,7)$ & $166(2,3)$ & $38(3,3)$ & \\
\hline OS, OSCIP ou ONG & $552(3,2)$ & $8(0,3)$ & $525(11,1)$ & $4(0,2)$ & $14(0,2)$ & $1(0,1)$ & \\
\hline Entidade filantrópica, empresa, cooperativa e outros & $1230(7,2)$ & $91(3,8)$ & $369(7,8)$ & $150(10)$ & $576(8,1)$ & $44(3,8)$ & \\
\hline Possui plano de carreira (respostas múltiplas) & $3501(21,0)$ & $981(40,4)$ & $1035(22,4)$ & $354(24,3)$ & $871(12,4)$ & $260(23,7)$ & $<0,001$ \\
\hline Progressão por antiguidade & $2885(87,9)$ & $861(91,3)$ & $842(85,7)$ & $296(90,0)$ & $679(85,6)$ & $207(88,1)$ & $<0,001$ \\
\hline Progressão por mérito & $1827(56,1)$ & $539(57,1)$ & $660(66,5)$ & $122(37,4)$ & $385(50,1)$ & $121(53,5)$ & $<0,001$ \\
\hline Progressão por titutlação e formação profissional & $2744(81,3)$ & $797(83,5)$ & $736(73,6)$ & $308(89,8)$ & $687(82,8)$ & $216(87,1)$ & $<0,001$ \\
\hline Incentivo, gratificação, prêmio financeiro por desempenho & $3966(23,4)$ & $654(26,7)$ & $1084(23,0)$ & $303(20,4)$ & $1740(24,4)$ & $185(16,2)$ & $<0,001$ \\
\hline
\end{tabular}


Tabela 2. Cirurgiões-dentistas participantes da avaliação externa do segundo ciclo do PMAQ-AB 2014, que possuem qualificação profissional em Especialização, Residência, Mestrado e Doutorado nas regiões do Brasil, 2013-2014

\begin{tabular}{|c|c|c|c|c|c|c|c|}
\hline \multirow{3}{*}{$\begin{array}{c}\text { Variável } \\
\text { (respostas múltiplas) }\end{array}$} & \multirow[b]{2}{*}{$\begin{array}{c}\text { Brasil } \\
(n=12514)\end{array}$} & \multicolumn{6}{|c|}{ Região } \\
\hline & & $\begin{array}{c}\text { Sul } \\
(\mathrm{n}=\mathbf{1 9 2 0})\end{array}$ & $\begin{array}{l}\text { Sudeste } \\
(n=3449)\end{array}$ & $\begin{array}{l}\text { Centro- } \\
\text { Oeste } \\
(n=1046)\end{array}$ & $\begin{array}{l}\text { Nordeste } \\
(n=5342)\end{array}$ & $\begin{array}{c}\text { Norte } \\
(n=757)\end{array}$ & \multirow[t]{2}{*}{ p-valor } \\
\hline & n $\quad(\%)$ & n (\%) & n (\%) & n $(\%)$ & n (\%) & n $(\%)$ & \\
\hline \multicolumn{8}{|l|}{ Especialização* } \\
\hline Saúde da Família & $3346(26,7)$ & $574(29,9)$ & $1335(38,7)$ & $255(24,4)$ & $1096(20,5)$ & $86(11,4)$ & $<0,001$ \\
\hline $\begin{array}{l}\text { Saúde Pública ou } \\
\text { Saúde Coletiva }\end{array}$ & $2339(18,7)$ & $400(20,8)$ & $739(21,4)$ & $176(16,8)$ & $911(17,1)$ & $113(14,9)$ & $<0,001$ \\
\hline Outra área & $6657(53,2)$ & $1085(56,5)$ & $1819(52,7)$ & $612(58,5)$ & $2692(50,4)$ & $449(59,3)$ & $<0,001$ \\
\hline \multicolumn{8}{|l|}{ Residência* } \\
\hline Saúde da Família & $200(1,6)$ & $50(2,6)$ & $84(2,4)$ & $8(0,8)$ & $53(1,0)$ & $5(0,7)$ & $<0,001$ \\
\hline $\begin{array}{l}\text { Saúde Pública ou } \\
\text { Saúde Coletiva }\end{array}$ & $121(1,0)$ & $28(1,5)$ & $45(1,3)$ & $13(1,2)$ & $34(0,6)$ & $1(0,1)$ & $<0,001$ \\
\hline Outra área & $256(2,0)$ & $40(2,1)$ & $112(3,2)$ & $20(1,9)$ & $69(1,3)$ & $15(2,0)$ & $<0,001$ \\
\hline \multicolumn{8}{|l|}{ Mestrado* } \\
\hline Saúde da Família & $48(0,4)$ & $12(0,6)$ & $20(0,6)$ & $2(0,2)$ & $11(0,2)$ & $3(0,4)$ & $<0,058$ \\
\hline $\begin{array}{l}\text { Saúde Pública ou } \\
\text { Saúde Coletiva }\end{array}$ & $146(1,2)$ & $32(1,7)$ & $50(1,4)$ & $8(0,8)$ & $46(0,9)$ & $10(1,3)$ & $<0,020$ \\
\hline Outra área & $899(7,2)$ & $137(7,1)$ & $278(8,1)$ & $76(7,3)$ & $345(6,5)$ & $63(8,3)$ & $<0,046$ \\
\hline \multicolumn{8}{|l|}{ Doutorado* } \\
\hline Saúde da Família & $33(0,3)$ & $4(0,2)$ & $10(0,3)$ & $2(0,2)$ & $15(0,3)$ & $2(0,3)$ & $<0,659$ \\
\hline $\begin{array}{l}\text { Saúde Pública ou } \\
\text { Saúde Coletiva }\end{array}$ & $35(0,3)$ & $9(0,5)$ & $11(0,3)$ & $4(0,4)$ & $10(0,2)$ & $1(0,1)$ & $<0,213$ \\
\hline Outra área & $207(1,7)$ & $29(1,5)$ & $79(2,3)$ & $19(1,8)$ & $66(1,2)$ & $14(1,8)$ & $<0,005$ \\
\hline
\end{tabular}

servidor público com a comunidade, segmentação do trabalho e interrupção nas ações de saúde, configuram grande desafio para a gestão do processo de trabalho das equipes de saúde ${ }^{14}$.

No presente estudo, o principal agente contratante foi a administração direta em todas as cinco regiões. Contudo existem diversas outras formas de contratação indicadas como: contratos temporários, contratos - Consolidação das Leis do Trabalho (CLT), cargos comissionados, autônomos, indicação e outros, apesar do MS configurar como trabalho precário o exercício desenvolvido sem a proteção social do trabalhador e prestado sem concurso público ${ }^{16}$ e considerar irregular todo vínculo de trabalho admitido na instituição pública sem concurso ou seleção de natureza pública, salvo exceções como trabalhadores terceirizados admitidos em áreas não finalísticas $^{17,18}$.

Em contrapartida, na região Sul, houve melhor desempenho na gestão do trabalho, o que contribui positivamente para a fixação dos 
Tabela 3. Distribuição dos tipos de ações e profissionais envolvidos com a Educação Permanente (EP) nas Equipes de Saúde Bucal (ESB) participantes da avaliação externa do segundo ciclo do PMAQ-AB 2014, segundo regiões do Brasil

\begin{tabular}{|c|c|c|c|c|c|c|c|}
\hline \multirow{3}{*}{ Variável } & \multirow[b]{2}{*}{$\underset{(n=17117)}{\text { Brasil }}$} & \multicolumn{6}{|c|}{ Região } \\
\hline & & $\underset{\substack{\text { Sul } \\
(\mathrm{n}=2478)}}{ }$ & $\begin{array}{c}\text { Sudeste } \\
(n=4762)\end{array}$ & $\begin{array}{c}\text { Centro- } \\
\text { Oeste } \\
(n=1509) \\
\end{array}$ & \multirow{2}{*}{$\begin{array}{c}\begin{array}{c}\text { Nordeste } \\
(\mathrm{n}=7204)\end{array} \\
\mathrm{n}(\%) \\
\end{array}$} & \multirow{2}{*}{$\begin{array}{c}\begin{array}{c}\text { Norte } \\
(\mathrm{n}=1164)\end{array} \\
\mathrm{n}(\%)\end{array}$} & \multirow[t]{2}{*}{ p-valor } \\
\hline & n $(\%)$ & n $(\%)$ & n $(\%)$ & n $(\%)$ & & & \\
\hline \multicolumn{8}{|c|}{ Ações e atividades de EP (respostas múltiplas) } \\
\hline $\begin{array}{l}\text { Seminários, mostras, } \\
\text { oficinas, grupos de } \\
\text { discussão }\end{array}$ & $10985(64,2)$ & $1750(70,6)$ & $3117(65,5)$ & $834(55,3)$ & $4661(64,7)$ & $623(53,5)$ & $<0,001$ \\
\hline Cursos presenciais & $8456(49,4)$ & $1604(64,7)$ & $2648(55,6)$ & $648(42,9)$ & $3179(44,1)$ & $377(32,4)$ & $<0,001$ \\
\hline Troca de experiência & $8028(46,9)$ & $1254(50,6)$ & $2570(54,0)$ & $536(35,5)$ & $3277(45,5)$ & $391(33,6)$ & $<0,001$ \\
\hline $\begin{array}{l}\text { Cursos de educação à } \\
\text { distância }\end{array}$ & $3813(22,3)$ & $740(29,9)$ & $1666(35,0)$ & $250(16,6)$ & $1056(14,7)$ & $101(8,7)$ & $<0,001$ \\
\hline Telessaúde & $3505(20,5)$ & $834(33,7)$ & $1373(28,8)$ & $334(22,1)$ & $857(11,9)$ & $107(9,2)$ & $<0,001$ \\
\hline UNASUS & $2373(13,9)$ & $599(24,2)$ & $812(17,1)$ & $122(8,1)$ & $779(10,8)$ & $61(5,2)$ & $<0,001$ \\
\hline $\begin{array}{l}\text { UBS como espaço de } \\
\text { formação (estagiários e } \\
\text { residentes) }\end{array}$ & $2831(16,5)$ & $487(17,6)$ & $836(17,6)$ & $173(11,5)$ & $1193(16,6)$ & $142(12,2)$ & $<0,001$ \\
\hline Tutoria/preceptoria & $1711(10,0)$ & $293(11,8)$ & $612(12,9)$ & $82(5,4)$ & $649(9,0)$ & $75(6,4)$ & $<0,001$ \\
\hline RUTE-telemedicina & $260(1,5)$ & $68(2,7)$ & $82(1,7)$ & $24(1,6)$ & $72(1,0)$ & $14(1,2)$ & $<0,001$ \\
\hline Outros & $1167(6,8)$ & $179(7,2)$ & $364(7,6)$ & $103(6,8)$ & $448(6,2)$ & $73(6,3)$ & $<0,035$ \\
\hline \multicolumn{8}{|c|}{ Profissionais envolvidos nas ações de EP } \\
\hline Toda a ESB & $11516(82,0)$ & $1883(84,4)$ & $3342(81,6)$ & $913(80,8)$ & $4729(82,0)$ & $649(80,2)$ & $<0,001$ \\
\hline $\begin{array}{l}\text { Apenas o cirurgião } \\
\text { dentista }\end{array}$ & $1973(14,1)$ & $271(12,1)$ & $630(15,4)$ & $163(14,4)$ & $796(13,8)$ & $113(14,0)$ & $<0,001$ \\
\hline $\begin{array}{l}\text { Apenas o Auxiliar e/ou } \\
\text { Técnico em Saúde } \\
\text { Bucal }\end{array}$ & $549(3,9)$ & $78(3,5)$ & $125(3,1)$ & $54(4,8)$ & $245(4,2)$ & $47(5,8)$ & $<0,001$ \\
\hline
\end{tabular}

Telemedicina $=$ Rede Universitária de Telemedicina.

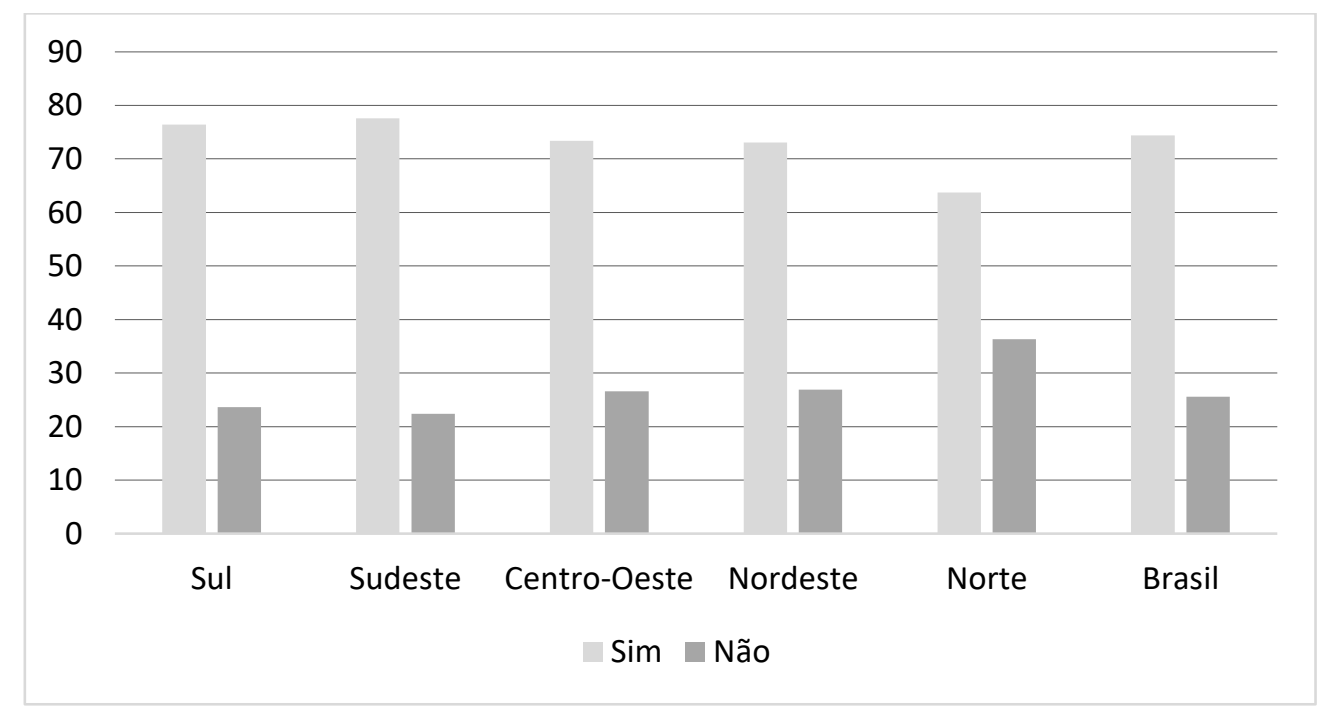

Figura 1. Distribuição da percepção sobre as ações de Educação Permanente contemplarem as demandas e necessidades das Equipes de Saúde Bucal participantes da avaliação externa do segundo ciclo do PMAQ-AB 2014, segundo regiões do Brasil $(\mathrm{p}<0,001)$ 
profissionais, e pode refletir em um cuidado mais longitudinal, como demonstrado em diversos indicadores de saúde ${ }^{19}$. No intuito de melhorar os vínculos de trabalho no SUS, o Governo Federal passou a incentivar a regularização das relações trabalhistas no setor público visando substituir os contratos precários por contratos legais através da realização de concursos públicos ${ }^{17}$, visto que a situação da gestão do trabalho atual ainda é uma grande barreira interposta ao alçance dos objetivos do SUS, sendo um dos principais motivos da alta rotatividade de profissionais na atenção básica ${ }^{7}$.

As desigualdades regionais também apresentaram o mesmo padrão sobre o baixo número de profissionais com plano de carreira, sendo o Sul a região com mais profissionais que disseram ter plano e o Nordeste a com menos. Um estudo realizado em 2008 apontou baixa frequência $(11,8 \%)$ de Plano de Cargos, Carreiras e Salários (PCCS) em capitais brasileiras e municípios com mais de 50 mil habitantes e mínimo de 500 postos de trabalho entre profissionais da saúde ${ }^{20}$. No presente estudo verificou-se maior prevalência de indicação de PCCS $(21,0 \%)$ e representa uma melhora significativa em uma década, considerando-se o fato de que, no caso do PMAQ$\mathrm{AB}$, houve a participação de municípios de pequeno porte, locais onde tem-se verificado menor proporção de $\mathrm{PCCS}^{21}$.

A progressão por antiguidade $\mathrm{e}$ titulação/formação profissional foram os mais prevalentes na pesquisa atual. Em outro estudo que avaliou experiências de sucesso de implantação de PCCS, os tipos de progressão considerados nos planos foram: avaliação de desempenho e qualificação profissional. No entanto, muitos PCCS não aderiram à progressão por antiguidade com receio do impacto financeiro que causaria ${ }^{22}$. Todos os planos de sucesso incluídos previam a avaliação do desempenho, mas nenhum dispunha de uma legislação que o regulamentava, sendo essa uma grande dificuldade e provavelmente a causa desse tipo de progressão ser o menos prevalente em âmbito nacional $^{22}$. Além disso, um estudo mostrou que grande parte dos municípios tem um plano único para todos os trabalhadores do município/estado ${ }^{21}$, embora os gestores considerem vantajoso tanto para a gestão como para os trabalhadores da saúde a existência de um plano específico, principalmente para valorização e fixação dos profissionais, refletindo na melhora da qualidade do serviço prestado à população ${ }^{20,23}$, inúmeras dificuldades são elencadas, de natureza financeira, legal, e técnica ${ }^{20}$ ou falta de uma política que contemple a sua implantação e a falta de autonomia da Secretaria Municipal de Saúde para criar o plano ${ }^{20,21}$.

Quanto à qualificação profissional dos $\mathrm{CD}$ na $\mathrm{AB}$, houve maior porcentagem de formação, seja em pós graduação do tipo latu sensu ou strictu sensu, em áreas odontológicas voltadas para práticas invidualistas, curativistas e isoladas em comparação com formação específica para Saúde da Família e/ou Saúde Pública/Coletiva. Esse aspecto pode reforçar ainda uma formação profissional na área voltada para especialidade e para uma abordagem em clínica privada e um processo de trabalho com prática isolada curativista, e pode interferir sobre a dificuldade em sua atuação na saúde pública ${ }^{24}$. Além disso, pode reforçar a ideia da atuação em serviço público com caráter generalista, e com baixa percepção sobre a necessidade de formação no campo da Saúde Coletiva. Um estudo mostrou que o preparo da equipe ao tema saúde da família foi um fator muito importante associado ao desempenho da Atenção Primária em Saúde, enaltecendo a importância e o valor da formação específica na área ${ }^{25}$, e o CD é, comparativamente aos médicos e enfermeiros, o profissional da $\mathrm{AB}$ com maior formação em especialização em Saúde da Família ${ }^{26}$.

No presente estudo, houve maior concentração de profissionais com formação em Saúde da Família ou Saúde Pública/Coletiva na 
região Sudeste e menor no Norte. Esse aspecto pode reforçar a desigualdade regional seja na distribuição dos profissionais, evidente na menor adesão de ESB no PMAQ-AB, como também na quantidade de cursos de graduação e especialização em cada região, com maior concentração no eixo sudeste-sul ${ }^{6}$. No entanto, a baixa qualificação ou preparo do profissional para atuação no contex to do serviço público pode limitar a qualidade da atenção e a melhoria do acesso da população aos serviços de saúde, pois precariza a capacidade de planejamento do trabalho e ações da equipe. Sendo assim, a especialização em Saúde da Família, pode gerar reflexões e mudanças no cotidiano dos trabalhadores como reorganização do processo de trabalho e desenvolvimento de atividades coletivas e de acolhimento à demanda espontânea ${ }^{12}$.

Além da especialização, deve-se reforçar a adesão à PNEPS visando mudança do processo de trabalho da equipe. No presente estudo, como na literatura $^{27}$, verificou-se maior acesso aos programas de EP presenciais. A principal limitação desses programas, é que as discussões ficam restritas ao pessoal da própria unidade de saúde privando a equipe de uma segunda opinião. $\mathrm{O}$ acréscimo de seus conhecimentos e atualização das ações e programas seriam desenvolvidos com o melhor uso de EP à distância, como o UNASUS, Telessaúde e RUTE-Telemedicina, abordagens que têm sido mais utilizadas por municípios de maior porte populacional ${ }^{27}$, e que no presente estudo foram mais utilizadas nas regiões Sul e Sudeste.

A EP, quando bem delineada, modifica o processo de trabalho, promove uma visão crítica nos profissionais, aumenta a participação social e aproxima a gestão das questões de saúde locais ${ }^{11}$. No entanto, um estudo com participantes do primeiro ciclo do PMAQ-AB mostrou que os profissionais relatam que algumas ações de EP não contemplam as demandas e necessidades da equipe $^{26}$, o que pode refletir sobre ações de EP com caráter convencional, definidas previamente por um comando central numa conjectura em que os profissionais não estavam se sentindo protagonistas do processo. Esses achados demonstram a necessidade de políticas efetivas de incentivo à adesão e capacitação dos profissionais dos municípios de pequeno porte, ao uso dos programas de EP à distância, contribuindo com sua formação e aumento na resolução da $\mathrm{AB}$.

No que diz respeito ao uso da Unidade Básica de Saúde (UBS) como espaço de formação para estagiários e residentes a média nacional foi $16,5 \%$, número muito reduzido no quadro geral brasileiro. Visto que o Brasil é um país com muitos cursos de graduação e pós-graduação em Odontologia, como citado anteriormente, esse número poderia ser melhorado significativamente com o aumento do número de UBS acolhendo estagiários e residentes em programas de formação. É dever do SUS, além do atendimento à população, garantir a formação de profissionais capacitados para o atendimento embasado nos princípios de universalidade e equidade, guiando-os a uma formação crítica com vistas ao desenvolvimento de valores humanísticos dos profissionais ${ }^{28}$. Outro ponto desanimador foi o número menor ainda de unidades que disponibilizam programas de tutoria/preceptoria, isso quer dizer que muitas unidades que recebem estagiários/residentes não possuem profissionais para supervisionar e orientar suas atividades.

Ao analisar os resultados, deve-se considerar que este estudo apresenta limitações por ser desenvolvido com dados secundários do PMAQ$\mathrm{AB}$, que pressupõe apenas a participação de equipes que participaram de forma espontânea e que se candidataram ao processo de avaliação. Assim, por não se tratar de uma amostra representativa de todas as ESB atuantes no país, podem não refletir o verdadeiro panorama da realidade da $\mathrm{AB}$ brasileira. No entanto, os mesmos merecem atenção por utilizarem dados abrangentes 
nacionais, e podem favorecer a compreensão da problemática estudada.

É notável no presente estudo uma boa qualificação profissional entre os $\mathrm{CD}$ participantes, no entanto, muito ainda pode ser feito para adequação da formação específica na área de Saúde Pública ou Saúde da Família. Além disso, pode-se verificar a abrangência alcançada pelos programas de EP, apesar de algumas regiões serem mais beneficiadas. Ao verificar a absorção de políticas, como fixação de profissionais, plano de carreira e implementação de políticas indutoras de EP na região Sul, percebe-se que é possível que outras regiões também alcancem o mesmo patamar, e políticas mais efetivas deveriam ser focadas nas demais regiões com os piores índices buscando um equilíbrio entre todas.

Apesar dos avanços, este estudo demonstrou que ainda há muitos desafios a serem enfrentados quanto aos recursos humanos no SUS. Precárias formas de seleção dos profissionais, baixa remuneração e baixa qualificação e formação dos funcionários para trabalhar com os problemas de saúde em âmbito coletivo, afetam de forma negativa o desenvolvimento de vínculos profissional-comunidade e o impacto no processo de trabalho e nas condições de saúde da população ${ }^{29}$.

\section{CONCLUSÃO}

A maioria dos CD participantes da avaliação externa do PMAQ-AB possuíam vínculos de trabalho considerados formais pelo MS, ingressaram no emprego via concurso público e foram contratados pela administração direta. Porém, a maioria dos profissionais não tem plano de carreira e os tipos de progressão prevalentes são por antiguidade e titulação/formação profissional.

Em relação à qualificação profissional, verificoupse maior prevalência de profissionais atuantes no SUS com especialidades odontológicas com foco em outras áreas que não a Saúde da
Família/Pública/Coletiva, fato que prejudica o processo de trabalho integrado da equipe e a forma de atenção e cuidado. A EP é mais desenvolvida em ações e cursos presenciais, onde os demais formatos, como as atividades à distância (Telessaúde, UNASUS e outras) não são muito utilizados e a integração ensino-serviço é incipiente, uma vez que poucas UBS recebem estudantes em sua formação.

Houve evidentes diferenças regionais relativas às questões analisadas com melhores indicadores na região Sul e Sudeste. Esse aspecto demonstrou que essas regioes foram capazes de absorver de políticas indutoras de desprecarização das relações trabalhistas, como fixação de profissionais, plano de carreira e de qualificação dos trabalhadores, com implementação de políticas de EP. No entanto, essas politicas precisam ser pensadas em uma forma de reduzir as desigualdades e as ações sejam incorporadas e efetivas nas demais regiões brasileiras.

\section{ABSTRACT \\ Regional inequalities in employment relationships and professional qualification of dentists working in Primary Care}

This transversal study aimed to compare employment relationships and professional qualification of dentists that work in Primary Care in different geographical regions in Brazil. Data was obtained from the Module VI of the external evaluation of the $2^{\text {nd }}$ Cycle of the Programa Nacional do Acesso e da Qualidade da Atenção Básica - PMAQ-AB (Primary Care Access and Quality National Program). The comparison between regions was carried out using the chi-square test $(\mathrm{p}<0.05)$ and descriptive analyses. In the 17,117 Oral Health Teams assessed, most of the dentists were statutory civil servants $(44.1 \%)$, and among them, most were found in the south region $(63.6 \%)$, while the lowest number was observed in the southeast $(37.8 \%)$. Nearly half of these professionals were approved in a public test (49.6\%), and the highest number was observed in the south $(79.5 \%)$, while 
the lowest number was found in the northeast (40.9\%). Regarding their professional qualification, $(73.1 \%)$ had complementary education, and among them, most were specialized in varied areas other than Collective Health (53.2\%), with the highest figures observed in the north $(59.3 \%)$ and the lowest in the northeast (50.4\%). Those specialized in Family Health or Collective/Public Health (26.7\% and $18.7 \%$, respectively) were most frequently found in the southeast $(38.7 \%$ and $21.4 \%$ ), while the lowest frequency was observed in the north $(11.4 \%$ and $14.9 \%$, respectively). In all comparisons, the " $p$ " value was $(p \leq 0.001)$. Therefore, regional inequalities were observed in employment relationships and professional qualification in Brazil, which reinforces the need for public policies that favor the retention and qualification of these professionals.

Descriptors: Oral Health. Health Personnel. Education, Professional.

\section{REFERÊNCIAS}

1. Spezzia $S$, Cavalheiro EM, Trindade LL. Uma análise das políticas públicas voltadas para os serviços de saúde bucal no Brasil. Rev Bras Odontol. 2015;72(1/2):109-13.

2. Brasil. Lei $n^{\circ} 8.080$ de 19 de Setembro de 1990 . Dispõe sobre as condições para a promoção, proteção e recuperação da saúde, à organização e o funcionamento dos serviços correspondentes e dá outras providências. Diário Oficial da União 1990; 19 set.

3. Brasil. Ministério da Saúde. Portaria n ${ }^{\circ}$ 1.444, de 28 de dezembro de 2000. Estabelece incentivo financeiro para a reorganização da atenção à saúde bucal prestada nos municípios por meio do Programa de Saúde da Família. Diário Oficial da União 2000; 28 dez.

4. Brasil. Ministério da Saúde. Política Nacional de Saúde Bucal. Brasília; 2004. [Acesso em 25 maio 2019]. Disponível em: https://aps.saude. gov.br/politicas/pnsb.

5. Ferreira NP, Ferreira AP, Freire MCM. Job market in dentistry: historical context and perspectives. Rev Odontol UNESP. 2013; 42(4):304-9.

6. Alissa SSM, Luiz AC, Stephani M, Letícia RMS, Ezequiel CR, Flávio FD. Distribuição dos cursos de Odontologia e de cirurgiões-dentistas no Brasil: uma visão do mercado de trabalho. Rev ABENO. 2018;18(1):63-73.

7. Carvalho M, Santos NR, Campos GWS. A construção do SUS e o planejamento da força de trabalho em saúde no Brasil: breve trajetória histórica. Saúde Debate. 2013;37 (98):372-87.

8. Batista KBC, Gonçalves OSJ. Formação dos profissionais de saúde para o SUS: significado e cuidado. Saúde Soc. 2011; 20(4):884-99.

9. Ceccim RB, Ferla AA. Educação Permanente em Saúde. 2009. [Acesso em 25 maio 2019]. Disponível em: http://www.sites.epsjv.fio cruz.br/dicionario/verbetes/edupersau.html\#topo/.

10. Seidl H, Vieira SP, Fausto MCR, Lima RCD, Gagno J. Gestão do trabalho na Atenção Básica em Saúde: uma análise a partir da perspectiva das equipes participantes do PMAQ-AB. Saúde Debate. 2014;38:94-108.

11. Yamamoto TS, Machado MTC, Silva Junior AG. Educação permanente em saúde como prática avaliativa amistosa à integralidade em Teresópolis. Trab Educ Saúde. 2015;13(3):61737.

12. Moreira KS, Lima CA, Vieira MA, Costa SM. Educação permanente e qualificação profissional para Atenção Básica. Saúde Pesq. 2017;10(1):101-9.

13. Cavalli LO, Rizzotto MLF, Guimarães ATB. O médico no processo de avaliação externa do Programa Nacional de Melhoria do Acesso e da Qualidade da Atenção Básica, ciclos I e II. Saúde Debate. 2016; 40(111):87-100.

14. Simas PRP, Pinto ICM. Trabalho em saúde: retrato dos agentes comunitários de saúde da região Nordeste do Brasil. Cien Saude Colet. 2017; 22(6):1865-76. 
15. Eberhardt LD, Carvalho M, Murofuse NT. Vínculos de trabalho no setor saúde: o cenário da precarização na macrorregião Oeste do Paraná. Saúde Debate. 2015;39 (104):18-29.

16. Bezerra MM, Medeiros KR. Limites do Programa de Melhoria do Acesso e da Qualidade da Atenção Básica (PMAQ-AB): em foco, a gestão do trabalho e a educação na saúde. Saúde Debate. 2018;42(2):188-202.

17. Mendonça MHM, Carsalade M, Giovanella L, Escorel S. Desafios para gestão do trabalho a partir de experiências exitosas de expansão da Estratégia de Saúde da Família. Cien Saude Colet. 2010; 15(5):2355-65.

18. Nogueira RP, Piola SF, Vianna SM. Problemas de gestão e regulação do trabalho no SUS. Serv Soc Soc. 2006; 87:147-62.

19. Paim J, Travassos C, Almeida C, Bahia L, Macinko J. The Brazilian health system: history, advances, and challenges. Lancet. 2011; 377(9779):1778-97.

20. Pierantoni CR, Varella TC, Santos MR, França T, Garcia AC. Gestão do trabalho e da educação em saúde: recursos humanos em duas décadas do SUS. Rev Saude Col. 2008; 18(4):685-704.

21. Magnago C, Pierantoni CR, França T, Vieira SP, Miranda RG, Nascimento DN. Política de Gestão do Trabalho e Educação em Saúde: a experiência do ProgeSUS. Cien Saude Colet. 2017; 22(5):1521-30.

22. Vieira SP, Pierantoni CR, Magnano C, França T, Miranda RG. Planos de carreira, cargos e salários no âmbito do Sistema Único de Saúde: além dos limites e testando possibilidades. Saúde Debate. 2017; 41(112):110-121.

23. Mendes TMC, Oliveira RFS, Mendonça JMN, Medeiros Junior A, Castro JL. Planos de Cargos, Carreiras e Salários: perspectivas de profissionais de saúde do Centro-Oeste do Brasil. Saúde Debate. 2018; 42(119):849-61.

24. Costa ICC, Araújo MNT. Definição do perfil de competências em saúde coletiva a partir da experiência de cirurgiões-dentistas atuantes no serviço público. Cienc Saude Colet. 2011; 16(1):1181-9.

25. Turci MA, Lima-Costa MF, Macinko J. Influência de fatores estruturais e organizacionais no desempenho da atenção primária à saúde em Belo Horizonte, Minas Gerais, Brasil, na avaliação de gestores e enfermeiros. Cad Saúde Pública. 2015; 31(9): 1941-52.

26. Rizzotto MLF, Gil CRR, Carvalho M, Fonseca ALN, Santos MF. Força de trabalho e gestão do trabalho em saúde: revelações da Avaliação Externa do Programa Nacional de Melhoria do Acesso e da Qualidade da Atenção Básica no Paraná. Saúde Debate. 2014; 38:237-51.

27. Baldani MH, Borges PKO, Ribeiro AE, Zermiani TC, Ditterich RG. A força de trabalho em saúde bucal na Atenção Primária à Saúde no estado do Paraná: um olhar sobre os resultados do PMAQAB. In: Ditterich RG, Graziani GF, Moysés SJ, organizadores Caminhos e trajetórias da saúde bucal no estado do Paraná. Londrina: INESCO; 2019. p. 127-149.

28. Sanseverino LM, Fonsêca GS, Silva TA, Junqueira SR, Zilbovicius C. Integração ensinoserviço na formação em Odontologia: percepções de servidores do Sistema Único de Saúde acerca da prática pedagógica no território. Rev ABENO. 2017; 17(3): 89-99.

29. Junqueira TS, Cotta RMM, Gomes RC, Silveira SFR, Batista RS, Pinheiro TMM, Sampaio RF. As relações laborais no âmbito da municipalização da gestão em saúde e os dilemas da relação expansão/precarização do trabalho no contexto do SUS. Cad Saúde Pública. 2010; 26(5):918-28.

\section{Correspondência para:}

Márcia Helena Baldani Pinto

e-mail: marciabaldani@gmail.com

Departamento de Odontologia

Campus Uvaranas, Bloco M, 52B, $2^{\circ}$ Piso

Av. General Carlos Cavalcanti, 4748 - Uvaranas 84030-900 Ponta Grossa/PR 